\title{
Effect of gauge boson mass on the phase structure of $\mathrm{QED}_{3}$
}

\author{
Jian-Feng Li ${ }^{1}$, Yu-Qing Zhou ${ }^{2}$, Hong-Tao Feng ${ }^{2}$, Wei-Min Sun ${ }^{1,3}$ and Hong-Shi Zong ${ }^{1,3}$ \\ 1 Department of Physics, Nanjing University, Nanjing 210093, China \\ 2 Department of Physics, Southeast University, Nanjing 211189 and \\ 3 Joint Center for Particle, Nuclear Physics and Cosmology, Nanjing 210093, China
}

\begin{abstract}
Dynamical chiral symmetry breaking (DCSB) in $\mathrm{QED}_{3}$ with finite gauge boson mass is studied in the framework of the rainbow approximation of Dyson-Schwinger equations. By adopting a simple gauge boson propagator ansatz at finite temperature, we first numerically solve the DysonSchwinger equation for the fermion self-energy to determine the chiral phase diagram of $\mathrm{QED}_{3}$ with finite gauge boson mass at finite chemical potential and finite temperature, then we study the effect of the finite gauge mass on the phase diagram of $\mathrm{QED}_{3}$. It is found that the gauge boson mass $m_{a}$ suppresses the occurrence of DCSB. The area of the region in the chiral phase diagram corresponding to DCSB phase decreases as the gauge boson mass $m_{a}$ increases. In particular, chiral symmetry gets restored when $m_{a}$ is above a certain critical value. In this paper, we use DCSB to describe the antiferromagnetic order and use the gauge boson mass to describe the superconducting order. Our results give qualitatively a physical picture on the competition and coexistence between antiferromagnetic order and superconducting orders in high temperature cuprate superconductors.
\end{abstract}

Key-words: $\mathrm{QED}_{3}$; DCSB, gauge boson mass

PACS Numbers: 11.10.Kk,11.15.Tk,11.30.Qc 
Quantum electrodynamics in $2+1$ dimensions $\left(\mathrm{QED}_{3}\right)$ has been widely studied in the last years. It is well known that $\mathrm{QED}_{3}$ has two basic features: dynamical chiral symmetry breaking (DCSB) in the zero fermion mass limit and confinement [1-8]. Moreover, $\mathrm{QED}_{3}$ is superrenormalizable, so it does not suffer from the ultraviolet divergence which are present in $\mathrm{QED}_{4}$. Therefore $\mathrm{QED}_{3}$ can be regarded as a useful tool by which one can develop insight into aspects of QCD. In parallel with a toy model of QCD, recently, as a low energy effective theory, $\mathrm{QED}_{3}$ is also of interest in $2 \mathrm{D}$ condensed matter systems, including high- $\mathrm{T}_{c}$ superconductors [9 16], quantum Hall effect [17] and graphene [18 21].

At zero temperature, by solving a truncated system of Dyson-Schwinger equations, Appelquist et al. first found that DCSB occurs only when the number of fermion flavors $N$ is less than a critical number $N_{c}[3,4]$. At finite temperature, Aitchison et al. adopted an approximate treatment of Dyson-Schwinger equation for the the fermion self-energy in the $1 / N$ expansion [22] and found that the critical number of fermion flavors $N_{c}$ is temperaturedependent and chiral symmetry is restored above a critical temperature. Recently, Feng et al [23 25] investigated the influence of finite chemical potential on the critical number of fermion flavors. It is interesting to study the influence of finite chemical potential and temperature on the critical number of fermion flavor simultaneously. In this paper we shall investigate the phase diagram of $\mathrm{QED}_{3}$ with gauge boson mass $m_{a}[26]$ at finite chemical potential and temperature. In particular, we study the effect of the gauge boson mass on phase structure of $\mathrm{QED}_{3}$. In addition, here we use DCSB to describe the antiferromagnetic (AF) order and use the gauge boson mass to describe the superconducting (SC) order [26, 27], and discuss the competition between $m_{a}$ and DCSB in chiral phase diagram of $\mathrm{QED}_{3}$ with gauge boson mass. We also note that the phase structure of $\mathrm{QED}_{3}$ is also useful for the study of semimetal-insulator transition in graphene.

In Euclidean space, the Lagrangian of $\mathrm{QED}_{3}$ with $N$ massless fermion flavors reads

$$
\mathcal{L}=\sum_{i=1}^{N} \bar{\psi}_{i}\left(\not \partial+\mathrm{i} e \not A-\gamma_{3} \mu\right) \psi_{i}+\frac{1}{4} F_{\rho \nu}^{2}+\frac{1}{2 \xi}\left(\partial_{\rho} A_{\rho}\right)^{2},
$$

where $\mathcal{L}$ contains the coupling between massless Dirac fermions and the $U(1)$ gauge field, and $\xi$ is the gauge parameter. The gauge boson can acquire a finite mass $m_{a}$ via the AndersonHiggs mechanism, which changes the original gauge boson propagator. The $4 \times 1$ spinor $\psi_{i}$ represents the fermion field, the $4 \times 4 \gamma_{\mu}$ matrices obey the Clifford algebra $\left\{\gamma_{\mu}, \gamma_{\nu}\right\}=2 \delta_{\mu \nu}$, and $i=1, \cdots, N$ are the flavor indices. For a real physical system, the number of fermion 
flavors $N$ equals to 2 (the realistic model corresponds to $N=2$, the two spin directions of the electron. see, for instance, [28]). So, in this paper we investigate the chiral phase diagram of $\mathrm{QED}_{3}$ with gauge boson mass $m_{a}$ at finite chemical potential and temperature for $N=2$ case. Adopting the rainbow approximation for the fermion-gauge vertex and neglecting the dependence of the gauge boson propagator on the chemical potential (this is a commonly used approximation in studying the dressed fermion propagator at finite $\mu$ [23 25, 30 37]), we can obtain the Dyson-Schwinger equation for the fermion propagator at finite chemical potential $\mu$ :

$$
S^{-1}(p, \mu)=i \gamma \cdot p-\mu \cdot \gamma_{3}+\frac{\alpha}{N} \int \frac{d^{3} k}{(2 \pi)^{3}} \gamma_{\rho} S(k, \mu) \gamma_{\nu} D_{\rho \nu}(q),
$$

where $q=p-k, \alpha=e^{2} N$, and $D_{\mu \nu}(q)$ is the gauge boson propagator in Landau gauge

$$
D_{\mu \nu}(q)=\frac{1}{q^{2}[1+\Pi(q)]+m_{a}^{2}}\left(\delta_{\mu \nu}-\frac{q_{\mu} q_{\nu}}{q^{2}}\right)
$$

with $\Pi(q)$ being the vacuum polarization function.

At non-zero chemical potential, according to the treatments in Refs. [23, 24], the inverse of the fermion propagator at finite $\mu$ can be obtained from the one at $\mu=0$ by the substitution $p \rightarrow \tilde{p}=\left(\vec{p}, p_{3}+i \mu\right):$

$$
S^{-1}(p, \mu)=S^{-1}(\tilde{p})=i \gamma \cdot \tilde{p} A\left(\tilde{p}^{2}\right)+m\left(\tilde{p}^{2}\right)
$$

where $S^{-1}(p)=i \gamma \cdot p A\left(p^{2}\right)+m\left(p^{2}\right)$ is the inverse of the fermion propagator at $\mu=0$. The validity of the above formula (4) has been discussed in detail in Ref. [38].

Now, let us give a short review of some studies on the effect of the wave function renormalization factor $A\left(p^{2}\right)$. At zero temperature and chemical potential, the results in Eq. (2) show that when the $1 / N$ order contribution to $A\left(p^{2}\right)$ is included, the critical fermion flavor number takes almost the same values as the case where $A\left(p^{2}\right)=1[4]$. At finite temperature temperature and zero chemical potential, a comparison of studies in Refs. [22, 39] also suggests that the $1 / N$ order contribution to $A\left(p^{2}\right)$ only slightly changes the results qualitatively. So, we expect that the the $1 / N$ order contribution to $A\left(p^{2}\right)$ is not important at finite temperature and chemical potential and we will take $A\left(p^{2}\right)=1$ in this paper. Then, we can obtain the integral equation for the dynamically generated mass function:

$$
m\left(\tilde{p}^{2}\right)=\frac{\alpha}{4 N} \int \frac{d^{3} k}{(2 \pi)^{3}} \operatorname{Tr}\left[\gamma_{\rho} \frac{-i \gamma \cdot \tilde{k}+m\left(\tilde{k}^{2}\right)}{\tilde{k}^{2}+m\left(\tilde{k}^{2}\right)} \gamma_{\nu} D_{\rho \nu}(q)\right]
$$


At non-zero temperature, for the purpose of obtaining a qualitative picture of dynamical picture of dynamical mass generation, we employ the following simplified gauge boson propagator ansatz [40, 41] in the presence of a finite gauge boson mass

$$
\Delta_{\mu \nu}\left(q_{0}, Q, \beta\right)=\frac{\delta_{\mu 3} \delta_{\nu 3}}{Q^{2}+\Pi_{0}(Q, \beta)+m_{a}^{2}},
$$

where $\Pi_{0}(Q, \beta)$ is defined as:

$$
\Pi_{0}(Q, \beta)=\frac{\alpha}{8 \beta}\left[Q \beta+\frac{16 \ln 2}{\pi} \exp \left(-\frac{\pi \beta}{16 \ln 2}\right)\right] .
$$

At finite temperature, the integration over the third component of a fermion loop momentum is replaced by an infinite sum over odd Matsubara frequencies. So the integral equation for the dynamically generated mass function at finite temperature and chemical potential is given by

$$
m(\beta, \mu, P)=\frac{\alpha}{N \beta} \sum_{n=-\infty}^{\infty} \int \frac{d^{2} k}{(2 \pi)^{2}} \frac{1}{Q^{2}+\Pi_{0}(\beta, Q)+m_{a}^{2}} \frac{m(\beta, \mu, K)}{\left[K^{2}+m^{2}(\beta, \mu, K)+\left(\varpi_{n}+i \mu\right)^{2}\right]},
$$

where

$$
\begin{array}{r}
p=\left(p_{0}, \mathbf{p}\right), P=|\mathbf{p}|, p_{0}=(2 m+1) \frac{\pi}{\beta}, \\
k=\left(k_{0}, \mathbf{k}\right), K=|\mathbf{k}|, k_{0}=(2 n+1) \frac{\pi}{\beta}, \\
q=\left(q_{0}, \mathbf{q}\right), Q=|\mathbf{q}|=|\mathbf{p}-\mathbf{k}|, q_{0}=2(m-n) \frac{\pi}{\beta} .
\end{array}
$$

The sum over infinite Matsubara frequencies can be done analytically:

$$
\begin{aligned}
& \sum_{n=-\infty}^{\infty} \frac{1}{\left(\varpi_{n}+i \mu\right)^{2}+K^{2}+m^{2}(\beta, \mu, K)} \\
= & \frac{1}{2 \sqrt{K^{2}+m^{2}(\beta, \mu, K)}} \times \sum_{n=-\infty}^{\infty}\left[\frac{1}{i \varpi_{n}+\sqrt{K^{2}+m^{2}(\beta, \mu, K)}}-\frac{1}{i \varpi_{n}-\sqrt{K^{2}+m^{2}(\beta, \mu, K)}}\right] \\
= & \frac{\beta}{2 \sqrt{K^{2}+m^{2}(\beta, \mu, K)}}\left[\frac{1}{e^{\beta}\left(\mu-\sqrt{K^{2}+m^{2}(\beta, \mu, K)}\right)+1}-\frac{1}{e^{\beta}\left(\mu+\sqrt{K^{2}+m^{2}(\beta, \mu, K)}\right)+1}\right],
\end{aligned}
$$

where we have made use of the formula

$$
\sum_{n=-\infty}^{\infty} \frac{1}{i \varpi_{n}-x}=\frac{\beta}{e^{\beta x}+1}
$$

Substituting Eq. (10) into Eq. (8), we can obtain the integral equation for the mass function with finite gauge boson mass at finite $T$ and $\mu$

$$
\begin{aligned}
& m(\beta, \mu, P)=\frac{\alpha}{8 N \pi^{2}} \int d^{2} K \frac{m(\beta, \mu, K)}{\sqrt{K^{2}+m^{2}(\beta, \mu, K)} \times\left[Q^{2}+\Pi_{0}(\beta, Q)+m_{a}^{2}\right]} \\
& \times\left[\frac{1}{e^{\beta}\left(\mu-\sqrt{K^{2}+m^{2}(\beta, \mu, K)}\right)+1}-\frac{1}{e^{\beta}\left(\mu+\sqrt{K^{2}+m^{2}(\beta, \mu, K)}\right)+1}\right] .
\end{aligned}
$$


Here we also note that in graphene system the gap equation has a similar form [21]:

$$
\begin{aligned}
m(\beta, \mu, P) & =\frac{\alpha}{8 N \pi^{2}} \int d^{2} K \frac{m(\beta, \mu, K) \times V(Q, \mu)}{\sqrt{K^{2}+m^{2}(\beta, \mu, K)}} \\
& \times\left[\frac{1}{e^{\beta}\left(\mu-\sqrt{K^{2}+m^{2}(\beta, \mu, K)}\right)+1}-\frac{1}{e^{\beta}\left(\mu+\sqrt{K^{2}+m^{2}(\beta, \mu, K)}\right)+1}\right],
\end{aligned}
$$

where the Coulomb interaction $V(Q, \mu)=\frac{1}{[Q / 8 \lambda+\Pi(\mu, Q) / N]}$ with the coupling strength $\lambda$ and the polarization function being given in Ref. [21]. In the context of graphene, the dominant interaction for Dirac fermions is the long-range Coulomb interaction, rather than gauge interaction. At finite chemical potential $\mu$, however, the polarization function is proportional to $\mu$ as $q$ tends to zero, which implies that the Coulomb interaction between Dirac fermions is statically screened and thus becomes short-ranged. The static screening factor $\mu$ appearing in the Coulomb interaction function in graphene system plays a similar role as the finite gauge boson mass $m_{a}$ in the effective $\mathrm{QED}_{3}$ theory of high- $T_{c}$ superconductor. For example, $\mu$ suppresses semimetal-insulator transition in graphene system, while $m_{a}$ suppressed DCSB in $\mathrm{QED}_{3}$ theory of high- $T_{c}$ superconductor.

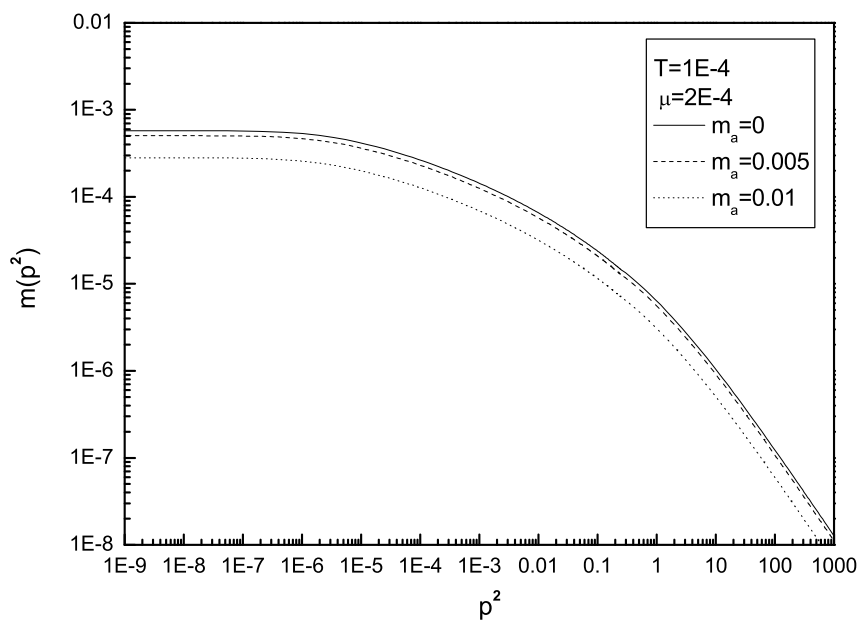

FIG. 1. $m\left(p^{2}\right)$ for several values of the gauge boson mass $m_{a}$ at $N=2, T=0.0001$ and $\mu=0.0002$.

Now we employ the iteration algorithm to numerically solve Eq. (12). In the numerical calculation we set $\alpha=1$, thus every quantity with mass dimension is scaled and becomes dimensionless. In the real numerical calculation, we adopt an ultraviolet cutoff $\Lambda=10^{5}$, which is large enough to ensure that the calculated results are stable with respect to $\Lambda$. The calculated dynamically generated mass function $m\left(p^{2}\right)$ is shown in Fig. 1 for $N=2$, 
$T=0.0001$ and $\mu=0.0002$, and for several values of the gauge boson mass. From Fig. 1 it can be seen that the dynamically generated mass $m\left(p^{2}\right)$ is almost constant for small $p^{2}$ and tends to zero at large $p^{2}$. This result is consistent with that given in Ref. [29]. In addition, we notice that $m\left(p^{2}\right)$ decreases with the gauge boson mass increasing for any fixed value of $p^{2}$. This reflects the following fact: Once the gauge boson acquires a finite mass via Anderson-Higgs mechanism, it cannot mediate a long-range interaction and suppresses the condensation of fermion-antifermion pairs.

The phase diagram of $\mathrm{QED}_{3}$ obtained in the presence of a small gauge boson mass for $N=2$ is plotted in Fig. 2. It is clearly seen that for each fixed value of the gauge boson mass the phase curve separates the DCSB phase (Nambu phase, where $m\left(p^{2}\right)>0$ ) and the chiral symmetric phase (Wigner phase, where $m\left(p^{2}\right)=0$ ). It can also be seen that the area of the region corresponding to the DCSB phase becomes small with $m_{a}$ increasing. This is understandable, because a finite gauge boson mass $m_{a}$ weakens the gauge interaction and suppresses the occurrence of DCSB.

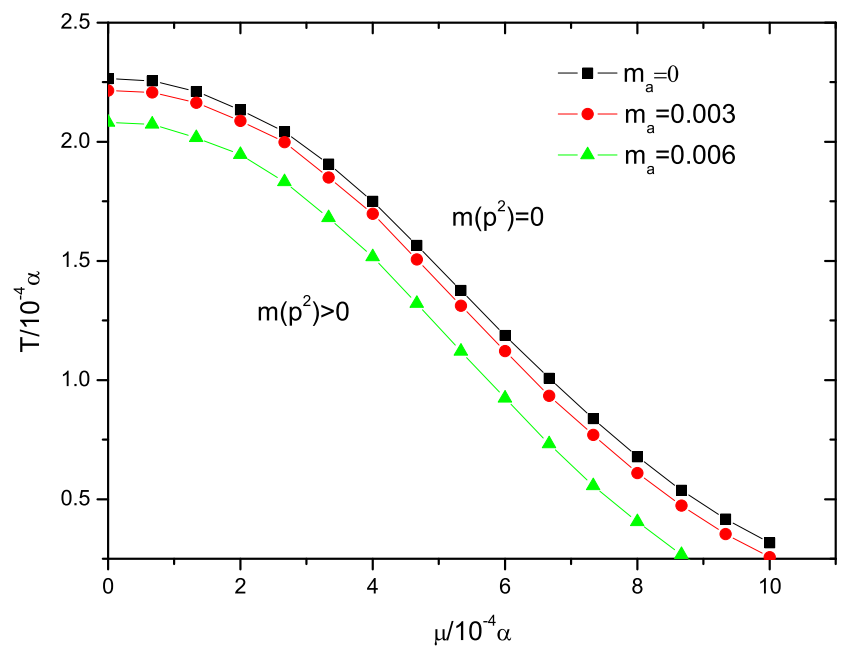

FIG. 2. The phase diagram of $\mathrm{QED}_{3}$ for several gauge boson mass $m_{a}$

From Fig. 2, it can be seen that when $m_{a}$ is above a critical value $m_{a}^{\text {crit }} \approx 0.018$, the DCSB phase will rapidly disappear on the $\mu-T$ plane. This result gives a simple physical picture on the competition between the $\mathrm{AF}$ order and the $\mathrm{SC}$ order in high temperature cuprate superconductors, where the AF order dominates at low doping while the SC order dominates at higher doping. In addition, from Fig. 2 it is easy to find that DCSB disappears 
and chiral symmetry gets restored when $\mu$ (or $T$ ) is above a certain critical value $\mu^{\text {crit }}$ (or $\left.T^{\text {crit }}\right)$, and the critical temperature decreases as the chemical potential $\mu$ increases.

Just as was shown above, in $\mathrm{QED}_{3}$ it is the dimensional coupling constant $\alpha$ (which is set to be 1 in our calculation) that sets the energy scale of the system. From our numerical results, it can be seen that the dynamically generated fermion mass, the critical temperature and chemical potential are quite small compared with this energy scale. Then, what is the reason for this? As far as we know, there is no simple way of seeing why the dynamically generated mass, the critical temperature and chemical potential are substantially less than the natural scale $\alpha$. However, this is what seems to happen in all such calculations. For example, in the BCS calculation of the superconducting energy gap at $T=0$, it is found that the zero-temperature gap, $\Delta$, is given by $\Delta \approx 2 \omega_{D} e^{-\frac{1}{V N_{F}}}$, where $\omega_{D}$ is the Debye (cutoff) frequency, $V$ is the volume and $N_{F}$ is the density of states. The quantity $V N_{F}$ plays the role of a dimensionless interaction parameter, and enters in a non-analytic way in the expression for $\Delta$. This is essentially because the calculation is (strongly) non-perturbative. The natural scale $\omega_{D}$ is substantially modified by the non-perturbative exponential factor (for details, see, for instance, Ref. [42]). This exponential factor multiplying the natural scale is very similar to what happens in the $T=0 \mathrm{QED}_{3}$ calculation. Something similar seems to be true at finite $T$ as well. The fact that such calculations lead to dynamically generated symmetry breaking masses which are substantially smaller than a natural energy scale might help to explain the "hierarchy problem", namely the wide discrepancy between (say) Grand Unification scales and the weak scale (for instance, the idea that $\mathrm{QED}_{3}$ might be a "laboratory" for studying the origin of different mass scales through non-perturbative dynamics was suggested in Ref. [43]).

It is well known that the cuprate superconductor is a Mott insulator with long-range AF order at half filling. Once holes are doped into the $\mathrm{CuO}_{2}$ planes, $\mathrm{SC}$ order occurs and the long-range AF order disappears in the material. Recently, elaborate neutron scattering and scanning tunneling microscopy experiments [44, 45] have found the competition between $\mathrm{AF}$ correlation and $\mathrm{SC}$ order in high- $T_{c}$ cuprate superconductor. It is clear that the gauge boson will become massive once SC appears in the cuprate superconductor. Here, in order to show the competition between the $\mathrm{AF}$ order and the $\mathrm{SC}$ order, following the approach of Ref. [27], we use the gauge boson mass to describe the SC order and use DCSB to describe the AF order. 
To summarize, in this paper, in the framework of the rainbow approximation of the Dyson-Schwinger equation and employing the approximation of ignoring the $\mu$ dependence of the photon propagator and setting the wave function renormalization factor $A\left(p^{2}\right)=$ 1 , we obtain the gap equation of $\mathrm{QED}_{3}$ at finite temperature and chemical potential. We investigate the phase diagram of $\mathrm{QED}_{3}$ for several values of gauge boson mass $m_{a}$

at finite chemical potential and temperature for $N=2$ case, which corresponds to real physical systems. In particular, we study the effect of a finite gauge boson mass on phase structure of $\mathrm{QED}_{3}$ and find that a finite gauge boss mass $m_{a}$ suppresses the DCSB, which shows a competition between antiferromagnetic order and superconducting orders in high temperature superconductors. Besides, by a comparison between Eq. (14) and Eq. (15), we expect that the excitionic semimetal-insulator transition in graphene can be well described by the phase structure of $\mathrm{QED}_{3}$.

\section{ACKNOWLEDGMENTS}

We are grateful to G.Z. Liu and I.J.R. Aitchison for helpful discussions. This work is supported in part by the National Natural Science Foundation of China (under Grant Nos. 10775069 and 10935001) and the Research Fund for the Doctoral Program of Higher Education (Grant No. 200802840009).

[1] J.M. Cornwall, Phys. Rev. D 22, 1452 (1980).

[2] R.D. Pisaski, Phys. Rev. D 29, 2423 (1984).

[3] T. Appelquist, M. Bowick, D. Karabali, and L.C.R. Wijewardhana, Phys. Rev. D 33, 3704 (1986).

[4] T. Appelquist, D. Nash, and L.C.R. Wijewardhana, Phys. Rev. Lett. 60, 2575 (1988).

[5] D. Nash, Phys. Rev. Lett. 62, 3024 (1989).

[6] P. Maris, Phys. Rev. D 52, 6087 (1995).

[7] P. Maris, Phys. Rev. D 54, 4049 (1996).

[8] A. Bashir, A. Raya, I.C. Cloët, and C.D. Roberts, Phys. Rev. C 78, 055201 (2008).

[9] P.A. Lee, N. Nagaosa, and C.G. Wen, Rev. Mod. Phys. 78, 17 (2006). 
[10] W. Rantner and X.G. Wen, Phys. Rev. Lett. 69, 1811 (1992).

[11] D.H. Kim, P.A. Lee and X.G. Wen, Phys. Rev. Lett. 76, 503 (1996).

[12] D.H. Kim and P.A. Lee, Ann. Phys. 272, 130 (1999).

[13] M. Franz and Z. TesAanovic, Phys. Rev. Lett. 87, 257003 (2001).

[14] I.F. Herbut, Phys. Rev. Lett. 88, 047006 (2002).

[15] I.F. Herbut, Phys. Rev. B 66, 094504 (2002).

[16] D.J. Lee and I.F. Herbut, Phys. Rev. B 66, 094512 (2005).

[17] X.G. Wen and A. Zee, Phys. Rev. Lett. 86, 3871 (2001).

[18] V.P. Gusynin, S.G. Sharapov et al, Phys. Rev. lett. 95, 146801 (2005).

[19] V.P. Gusynin, S.G. Sharapov et al, Phys. Rev. lett. 96, 256802 (2006).

[20] D.V. Khveshchenko, Phys. Rev. lett. 87, 246802 (2001).

[21] G.Z. Liu, W. Li, and G. Cheng, Phys. Rev. B 79, 205429 (2009).

[22] I.J.R. Aitchison, N. Dorey et al, Phys. Lett. B 294, 91 (1992).

[23] H.T. Feng, F.Y. Hou, X. He, W.M. Sun, and H.S. Zong, Phys. Rev. D 73, 016004 (2006).

[24] H.T. Feng, W.M. Sun, D.K. He, and H.S. Zong, Phys. Lett. B 661, 57 (2008).

[25] H.T. Feng, M. He, W.M. Sun, and H.S. Zong, Phys. Lett. B 688, 178 (2010).

[26] T.P. Barnea and M.Franz, Phys. Rev. B 67, 060503 (2003).

[27] G.Z. Liu and G. Cheng, Phys. Rev. D 67, 065010 (2003); G.Z. liu and G. Cheng, arXiv:cond-mat/0208061

[28] J.B. Marston and I. Affleck, Phys. Rev. B 39, 11538 (1989).

[29] M. He, H.T .Feng, W.M. Sun, and H.S. Zong, Mod. Phys. Lett. A 22, 449 (2007).

[30] C.D. Roberts and S.M. Schmidt, Prog. Part. Nucl. Phys. 45S1, 1 (2000), and references therein.

[31] H.S. Zong, L. Chang, F.Y. Hou, W.M. Sun, and Y.X. Liu, Phys. Rev. C 71, 015205 (2005).

[32] F.Y. Hou, L. Chang, W.M. Sun, H.S. Zong, and Y.X. Liu, Phys. Rev. C 72, 034901 (2005).

[33] Y. Taniguchi and Y. Yoshida, Phys. Rev. D 55, 2283 (1997).

[34] D. Blaschke, C.D. Roberts, and S. Schmidt, Phys. Lett. B 425, 232 (1998).

[35] P. Maris, C.D. Roberts, and P.C. Tandy, Phys. Lett. B 420, 267 (1998).

[36] A. Bender, W. Detmold, and A.W. Thomas, Phys. Lett. B 516, 54 (2001).

[37] O. Miyamura, S. Choe, Y. Liu, T. Takaishi, and A. Nakamura, Phys. Rev. D 66, 077502 (2002). 
[38] Y. Jiang, Y.M. Shi, H.T. Feng, W.M. Sun, and H.S. Zong, Phys. Rev. C 78, 025214 (2008).

[39] I.J.R. Aitchison and M. Klein-Kreisler, Phys. Rev. D 50, 1068 (1994).

[40] N. Dorey and N.E. Mavromatos, Nucl. Phys. B 386, 614 (1992).

[41] N. Dorey and N.E. Mavromatos, Phys. Lett. B 266, 163 (1991).

[42] I.J.R. Aitchison and A.J.G. Hey, Gauge Theories in Particle Physics, Vol. 2 (Bristol and Philadelphia: IoP Publishing, 2004), section 17.7.

[43] M.R. Pennington and D. Walsh, Phys. Lett. B 253, 246 (1991).

[44] B. Lake et al., Science. 291, 1759 (2001); Nature (London). 415, 299 (2002).

[45] J.E. Hoffman et al., Science 295, 466 (2002). 